\title{
Rapid, low-cost patterning of microstructures in polydimethylsiloxane via mask-less laser-machining
}

\author{
C. L. Sones, I. N. Katis, B. Mills, M. Feinaugle, A. Mosayyebi, J. Butement, R. W. Eason \\ Optoelectronics Research Centre, University of Southampton, Highfield, Southampton, SO17 1BJ, U.K
}

Polydimethylsiloxane (PDMS), due to its unique characteristics including biocompatibility, elasticity (which allows it to be flexibly moulded into the desired shape), optical transparency, and its low-cost provides a valuable advantage as a building material for the fabrication of microfluidics-based lab-on-chip devices and micro-contact printing [1, 2] moulds that allow parallel deposition of various materials on a target surface. A range of methodologies such as wet chemical etching, dry plasma etching, decal transfer microlithography, and bond-detach method have been utilised for creating patterns in PDMS. However, one of the most commonly used approaches for the prototyping of PDMS for such applications is soft-lithography. This involves the use of a clean-room based UVlithography step that uses expensive custom-designed masks for the fabrication of a master-mould with structures that are then duplicated via stamping of this master to produce a secondary-mould in PDMS, which is then used for micro-contact printing applications. Even though this lithographic procedure can routinely produce high-resolution micron-scale structures, the procedure is time-consuming and expensive. Instead, for the production of the master, we propose as a cheap alternative to expensive UV-lithography, a mask-less laser-based procedure which does not rely on cleanroom access. Similar to the soft-lithographic procedure, the process is two-step and allows the creation of high-quality sub-micron to millimetre-scale features, in a wide range of materials, with the added advantage of being able to fabricate complex and differently-shaped structures adjacent to each other, in either a sequential or a single-step. This laser-based method (Fig.1) has been used to create two-dimensional surface relief patterns in a master-mould for replication into PDMS and subsequent contact-printing.

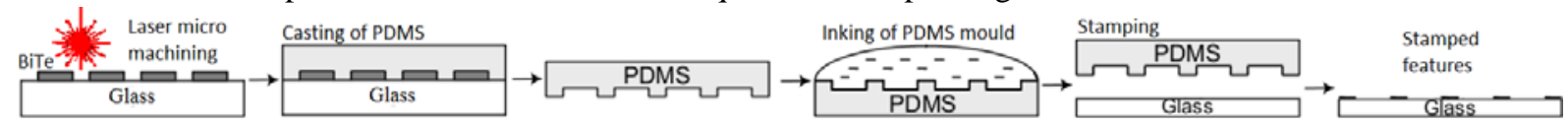

Fig. 1 Schematic of the laser-based contact-printing procedure.

The desired patterns were first created in a bismuth telluride (BiTe) film ( $1 \mu$ thick) sputtered onto a glass substrate, for the creation of a master. BiTe films were used for this, as their ablation properties were particularly good, but any material that can be similarly ablated to imprint the primary patterns could be used. The BiTe film was patterned using ultra-short laser pulses $(150 \mathrm{fs}, 800 \mathrm{~nm})$ that had spatially modified intensity profiles produced via diffraction from a digital micro-mirror device to create a two-dimensional array of $10 \times 10$ square trenches that were $30 \mu \mathrm{m} \times 30 \mu \mathrm{m}$ in their dimensions and had a centre-to-centre separation of $\sim 50 \mu \mathrm{m}$. Each square trench was ablated in the BiTe film using 10 sequential laser pulses with intensity of $1.7 \mathrm{~J} / \mathrm{cm}^{2}$. Individual features with varied shapes and sizes can be ablated next to one another with simple modification of the projected image pattern. The ablated square pattern was then used to form a mould of PDMS. A 10:1 mixture of a PDMS base and a curing agent was weighed in the right proportion, then mixed thoroughly and poured onto the patterned BiTe film. The mixture was allowed to set at $90^{\circ} \mathrm{C}$ for at least one hour. As shown in Fig.2a, this led to the creation of a complementaryshaped micron-scale two-dimensional pattern of upstanding squares in PDMS. To demonstrate the usefulness of this PDMS structuring approach for micro-contact printing, we used the PDMS-mould to contact print an ink pattern onto a glass substrate. Fig.2b shows the ink pattern micro-contact printed using the PDMS master pre-fabricated using the laser-based machining method described above.
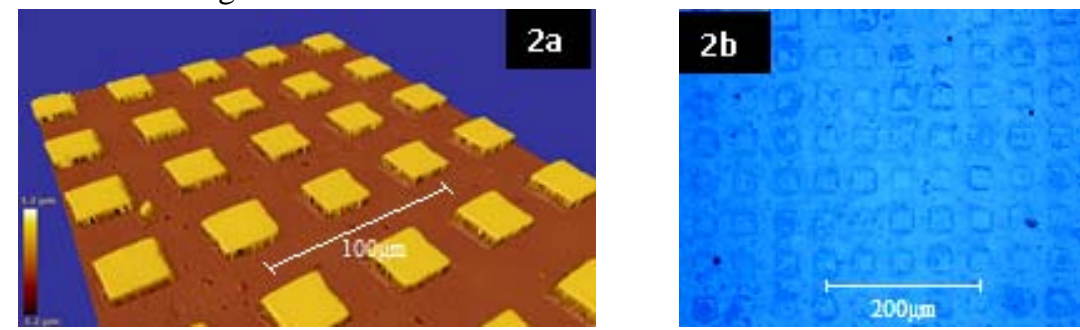

Fig. 2 (a) Interference-based microscope image of formed patterns in PDMS; (b) Optical microscope image showing features contact-printed using the same pattern.

We believe this laser-based approach can be extended to form any complex micron and submicron scale structures that are not only useful in contact printing applications but also in the fabrication of millimetre scale labon-chip fluidic devices.

\section{References}

[1] A. Kumar, G. M. Whitesides," Features of Gold having micrometer to centimetre dimensions can be formed through a combination of stamping with an elastomeric stamp and an Alkanethiol ink followed by chemical etching," Appl. Phys. Lett. 63(14), 2002, (1993).

[2] A. Bernard, J. P. Renault, B. Michel, H. R. Bosshard, E. Delamarche, “Microcontact Printing of Proteins," Adv. Mater.12(14), 1067, (2000). 